\title{
Horta Compartilhada com IOTA e Fuzzy - Um Projeto de Economia das Coisas para IoT
}

\author{
Fábio Carli R. Teixeira ${ }^{1}$, Jonas L. de Vilas Boas ${ }^{1}$, Antônio M. Alberti ${ }^{1}$
}

Resumo - Utilizando um cenário de uma horta comunitária urbana, este artigo apresenta a aplicação da Internet das Coisas em um caso de Economia Compartilhada, fazendo uso da criptomoeda IOTA, seu protocolo de registros distribuídos e da lógica fuzzy, de forma a se ter o controle das funcionalidades da horta, seus pagamentos, a distribuição de produtos, e o monitoramento do desenvolvimento das plantas. A horta pode ser acomodada em um edifício, garagem e até mesmo em um terreno baldio, de forma a otimizar e a escalar o aproveitamento dos recursos utilizados e produtos oferecidos.

Palavras-Chave - Economia das Coisas, IoT, Horta, Compartilhada.

Abstract - Using a shared vegetable garden scenario, this article presents the application of the Internet of Things in a case of Shared Economy, using the IOTA cryptocurrency and its distributed records protocol, and the fuzzy logic, in order to have control over the functionalities of the vegetable garden, their payments, the distribution of products, and the monitoring of the development of the plants. The vegetable garden can be accommodated in a building, garage and even in a vacant lot, in order to optimize and scale the use of resources used and products offered.

\section{Keywords - Economy of Things, IoT, Vegetable Garden, Shared.}

\section{INTRODUÇÃO}

Hortas comunitárias são feitas para uso coletivo, por um grupo de pessoas associadas. Em geral, o cultivo é feito por todos da comunidade, sendo os produtos divididos de forma igual entre todos, e o excedente comercializado ou trocado por outros produtos. Mas a participação nos trabalhos de cultivo nem sempre é possível para todos, de forma que alguns membros da comunidade administrem e operem a horta, e outros colaborem financeiramente, e ainda assim todos participem na divisão dos produtos. Outra questão é o local de cultivo, feito preferencialmente em um ambiente aberto e com iluminação natural, ou em estufas e até mesmo em ambientes prediais internos, como é o caso de muitas hortas que utilizam áreas de garagem de prédios, com iluminação artificial que simula o espectro da luz solar. A irrigação é outro aspecto importante de ser monitorado, e varia com o tipo de planta, de solo, de luminosidade etc. Atualmente o sensoriamento das condições de solo, umidade, clima, luminosidade, podem ser eficientemente realizados e o desenvolvimento das plantas pode ser aprimorado, ainda que não se use venenos e insumos químicos no cultivo.

Este artigo apresenta uma proposta de Economia das Coisas para Horta Comunitária Urbana. São utilizadas tecnologias que se adequem a este cenário, tal como a criptomoeda IOTA para os micropagamentos, software de monitoramento das funções da horta com lógica fuzzy para tomar as decisões das funções de controle da horta e todos os equipamentos necessários.

${ }^{1}$ Instituto Nacional de Telecomunicações (INATEL) - ICT Lab. Av. João de Camargo, 510 - Centro, Santa Rita do Sapucaí, MG, 37540-000. fabio.carli@mtel.inatel.br, jonas.lopes@dtel.inatel.br, alberti@inatel.br
A IoT tem transformado a Internet em uma rede sensorial (umidade, iluminação, temperatura, etc.), e a adoção de um ativo digital, para monetizar as trocas de dados e produtos em uma aplicação de IoT, permite o surgimento de um novo ambiente colaborativo, que permite o compartilhamento entre os atores do processo, distribuindo as responsabilidades e os benefícios à coletividade. Com técnicas de inteligência artificial, como a lógica fuzzy, os dados gerados por dispositivos IoT podem ser processados, melhorando o controle dos sistemas e otimizando os processos. Com isso, é possível expandir os conceitos da horta apresentados para se pensar no uso da IoT na economia compartilhada de uma forma geral, possibilitando o compartilhamento de bens e serviços de uma comunidade, gerando novas formas de negócios e melhor atendendo as necessidades dos usuários.

Naturalmente, o uso da moeda digital ainda elenca muitas dúvidas, principalmente no que se refere à garantia e à segurança da transação. É uma forma de pagamentos revolucionária que se propõe a substituir as transações financeiras convencionais [1]. Neste trabalho, serão descritas as características da moeda digital IOTA, a mais adequada aos micropagamentos de serviços de IoT, por não cobrar taxas, e por sua alta escalabilidade. $\mathrm{Na}$ Seção II será feita uma exposição do funcionamento básico da rede Tangle, que é a rede onde as transações são efetivadas e validadas. Na seção III são tecidos breves comentários sobre a lógica fuzzy, utilizada para a tomada de decisões. A Seção IV mostra a integração do protocolo IOTA, da lógica fuzzy e das funcionalidades da horta. A Seção V apresenta uma análise preliminar de desempenho da solução implementada. Finalmente, a Seção VI conclui o artigo e apresenta possíveis trabalhos futuros.

\section{O PROTOCOLO IOTA}

A moeda digital IOTA inicialmente aborda duas questões: a escalabilidade e as taxas cobradas pelas transações, que são problemas decorrentes do uso de Blockchain com PoW (Proofof-Work - Prova de Trabalho). A PoW é realizada pelos Nós da rede para validar e garantir o consenso das transações [2]. Em IOTA é introduzido um novo conceito: o Tangle [3] [4], que é um Grafo Acíclico Dirigido (DAG - Directed Acyclic Graph), com registros distribuídos na rede, sem mineradores e tampouco Blockchain. Como não há taxas de mineração no Tangle, fica mais fácil fazer micro e até nano pagamentos entre dispositivos IoT (Internet of Things), uma das razões da existência desta moeda digital, e da adoção deste protocolo no projeto da horta. Desta forma, a IOTA é uma tecnologia totalmente diferente da maioria das criptomoedas existentes [6], solucionando algumas limitações da Blockchain, tais como as altas taxas de mineração cobradas, o custo energético de manter a rede funcionando e o tempo de geração e confirmação de blocos. Além da grande escalabilidade (e velocidade), ela é resistente a ataques por computação quântica e possui estrutura modular e leve, própria para os pequenos dispositivos de IoT. Como não há nós mineradores na IOTA, todos os nós têm igual poder, e o 
mecanismo de consenso ocorre em cada uma das transações realizadas, garantindo maior eficiência.

Também dispensa a necessidade de backups, pois não há arquivos que possam ser perdidos. Basta que se tenha uma senha guardada de forma segura. Se na Blockchain quanto mais transações ocorrerem, mais lenta e mais caras as tarifas, na IOTA a rede fica cada vez mais rápida, pois cada transação em sua rede faz a confirmação de consenso de outras transações. Como as micro e nano transações serão maioria na IoT, é vantajoso para a rede IOTA fazê-las.

O protocolo IOTA é um protocolo criado especialmente para transações entre objetos na IoT [7]. O Tangle é a tecnologia DLT (Distributed Ledger Technology) da IOTA, permitindo, além do que já foi citado, que não ocorram forks, garantindo a imutabilidade da informação e maior segurança nas transações. O fork é um tipo de bifurcação, que acontece quando não há um consenso entre os nós da rede. A divergência pode provocar uma bifurcação na cadeia de blocos, e os usuários precisam decidir qual cadeia continuarão seguindo. Isso não acontece em IOTA.

O Tangle é feito de Sites e nós. Os Sites contêm uma ou algumas transações relacionadas entre si, e os nós são os usuários IOTA que emitem e recebem as transações. Todos os nós da rede são responsáveis por aprovar as transações, o que remove o papel dos mineradores na rede.

A primeira transação que ocorreu no Tangle foi chamada de Genesis. Ela é direta ou indiretamente aprovada por todas as outras subsequentes. Esta transação envia tokens de um endereço contendo todos os tokens para outros endereços do fundador. No estágio inicial, não se escolhem as transações para aprovação. Todos os nós seguem algumas regras de referência, pois geralmente são dispositivos locais que pertencem a uma mesma região. A transação recém-emitida, que ainda não recebeu nenhuma aprovação é denominada TIP. Há um algoritmo de seleção de TIP, que resolve os conflitos de aprovações pendentes. Este algoritmo roda diversas vezes para verificar qual das TIPs tem maior probabilidade de ser aprovada pelo nó. A nova transação emitida por um usuário deve sempre primeiramente aprovar duas TIPs existentes, e isso pode ser feito de forma quase aleatória, uma vez que o algoritmo decide a melhor TIP a ser aprovada, evitando nós preguiçosos, que poderiam sempre aprovar um par fixo de transações antigas, e também evitando que entidades maliciosas inflem artificialmente o número de TIPs, tornando possível que transações futuras selecionem as TIPs maliciosas e abandonem as TIPs honestas. O Tangle atua de forma assíncrona e tolera duas transações conflitantes, pois supõe que a transação incorreta será considerada órfã ou até mesmo apagada, à medida que o Tangle for crescendo. Ainda vale observar que um nó será descartado pelo nó vizinho, quando mostrar preguiça em relação à propagação de transações. O incentivo mantém todos os nós funcionando, embora eles não emitam transações frequentes. Popov [3] considera que a estratégia de aprovação das TIPs é o fator mais importante na construção da criptomoeda baseada em Tangle, pois é lá que a maioria dos ataques estarão escondidos.

\section{A. Gerando Consenso na Rede}

Ao se fazer uma transferência entre duas carteiras IOTA, um certo tempo será necessário para que a rede aceite essa transação como válida e adicione o valor da transação ao saldo da carteira receptora. Para se obter a aprovação da transação pela maioria das transações subsequentes, e assim obter o consenso na rede, há uma forma lógica de processamento das transações no Tangle. Transações distintas possuem pesos distintos. Quanto mais produtivo for um nó da rede, mais peso terão as transações que por ele passarem. E quanto mais aprovações forem recebidas por uma transação, maior também será o peso que ela carrega. Dessa forma, as transações corretas, consideradas válidas, receberão mais atenção dos nós que as incorretas (inválidas), uma vez que a transação correta irá ser acelerada no Tangle pelo seu aumento de peso, enquanto as incorretas ficarão cada vez mais lentas. E à medida em que uma transação for aprovada, com outras transações aprovadas em seu histórico, ela receberá parte do peso dessas outras transações, e assim, quanto mais transações válidas em contato umas com as outras, mais elas serão aceleradas no Tangle. Essa ponderação que o Tangle promove nas transações gerencia o rendimento da rede, e evita, ou minimiza, os ataques de spam. Assim, a capacidade dos nós de processar transações de um cenário específico fica limitada, mas a segurança é preservada.

Em um regime de carga baixa, a transação, após aprovada várias vezes pelas transações subsequentes, terá seu peso acumulado crescendo de forma constante, em uma velocidade proporcional ao peso médio de uma transação genérica da rede. Em carga alta, após um período de adaptação, onde o peso acumulado cresce de forma lenta, a velocidade de crescimento desse peso irá aumentar de forma similar ao regime de carga baixa. Esse período de adaptação é o tempo que a transação aguarda para ser aprovada pela maioria das TIPs atuais. A Figura 1 ilustra esse mecanismo:

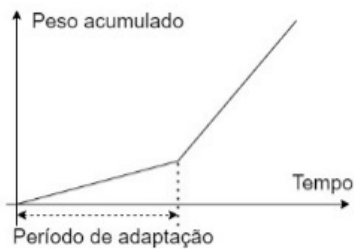

Fig. 1. Crescimento do Peso acumulado em carga alta [3].

\section{LÓGICA FUZZY}

O sistema lógico tradicional considera respostas determinísticas com base em regras formais. No entanto, no mundo real uma tomada de decisão mais flexível é usual. Os humanos têm capacidade de tomar decisões racionais até mesmo com incertezas e imprecisões, considerando o conhecimento adquirido previamente. A lógica fuzzy visa modelar este comportamento e permitir suposições aproximadas com base em probabilidade, delimitando as respostas possíveis com base na lógica clássica [5].

Em um sistema fuzzy, as entradas determinísticas são mapeadas em conjuntos fuzzy. Os valores probabilísticos são inferidos com base em uma função de pertinência e as decisões são tomadas com base nas regras aplicadas sobre esses conjuntos. Para cada variável de entrada, o sistema fuzzy define predicados, como "alto", "médio" e "baixo" ou "muito frio", "frio", "agradável", "morno" e "quente". É definido um intervalo de valores para cada predicado podendo haver sobreposição entre os predicados, ou seja, algum intervalo de valores pode ser descrito em mais de um predicado. Além disso, regras de inferência são definidas, com base no conhecimento de especialistas ou algoritmos de aprendizado de máquina, para restringir as entradas. Os valores fuzzy são decodificados em valores úteis nas aplicações reais, também chamados valores crisp, e retornados como saídas do sistema. 


\section{PROPOSTA E IMPLEMENTAÇÃO DA HORTA}

A discussão apresentada na Seção II tem sua importância no sentido de se entender em quanto tempo, após efetuada a transferência da carteira emissora para a carteira receptora, se verificará a alteração de saldo nesta última. No caso da horta aqui apresentada, o sistema de irrigação e iluminação artificial será acionado mediante a transferência de IOTAs. É um princípio para se criar a Economia das Coisas de um ambiente de IoT. Para verificar as condições da horta foram utilizados sensores de umidade do solo nos canteiros e um sensor de umidade e temperatura do ar.

A placa Raspberry Pi 3, é a base de hardware deste sistema, e deverá estar equipada com um cartão SD compatível. O sistema operacional a ser utilizado é a distribuição Linux Raspbian, que é o SO oficial da Fundação Raspberry. As instruções de instalação estão detalhadas no site da Fundação [8]. A linguagem Python, a ser utilizada, já está pré-instalada no Raspbian. Para que se tenha acesso ao Tangle da IOTA, é necessário que se instale a biblioteca API PyOTA, que está disponível no GitHub [9]. Diversos códigos Python relacionados a IOTA podem ser instalados e executados na Raspberry Pi. No próprio GitHub [10] há vários exemplos diferentes para se executar, nesse controle de horta inteligente foi usada uma adaptação desses códigos.

Visando melhorar a produtividade das plantas e utilizar o saldo depositado eficientemente, foi desenvolvido um controlador inteligente da irrigação utilizando a lógica fuzzy. $\mathrm{O}$ controlador recebe as condições da horta e o valor do saldo decide por quanto tempo deve acionar a bomba de irrigação. Algumas soluções propostas na literatura aplicando a lógica fuzzy consideram a temperatura do ambiente e a umidade do ar [12] [13]. A planta escolhida para este trabalho foi o orégano, a fim de ajustar o sistema para tomar decisões mais precisas. Todas as regras do sistema podem ser ajustadas de acordo com a variedade de hortaliças produzidas. $\mathrm{O}$ ambiente de cultivo se assemelha a um ambiente doméstico, ou seja, um ambiente interno com pouca ou nenhuma exposição ao sol. As plantas de orégano se ajustam melhor com solo úmido, consumindo cerca de $2001 / \mathrm{m}^{2}$ ao ano de água no cultivo. A temperatura do ambiente adequada pode variar de $4^{\circ} \mathrm{C}$ a $32^{\circ} \mathrm{C}$, respondendo melhor a temperaturas entre $21^{\circ} \mathrm{C}$ e $25^{\circ} \mathrm{C}$, e a umidade do ar ideal está entre $60 \%$ e $65 \%$.

Outra condição particular desse trabalho que deve ser considerada é o valor do saldo. A horta será mantida com depósitos realizados pelas pessoas da comunidade. Por isso, o saldo também deve ser analisado no momento do controle da irrigação. Foi considerado que cada unidade do saldo corresponde a um segundo de funcionamento da bomba, que tem uma vazão de $20 \mathrm{ml} / \mathrm{s}$. Para o cenário proposto, existem três vasos com plantas de orégano de $0,375 \mathrm{~m}^{2}$, consumindo um total de $200 \mathrm{ml}$ de água por dia. Dessa forma, seria necessário um saldo de cerca 70 IOTAs por semana para irrigar a horta.

O estado da horta será verificado a cada 6 horas. O sistema ficará em estado suspenso a maior parte do tempo para economizar energia. Se houver saldo disponível, a iluminação será mantida ligada durante um desses intervalo, totalizando 6 horas de exposição à iluminação artificial. A iluminação não decrementa o saldo, mas não será acionada se o saldo for nulo. Em cada intervalo as condições serão verificadas e o sistema decidirá por quanto tempo irá acionar a bomba para irrigar as plantas. A Figura 2 mostra o fluxograma do sistema.

Foram definidas as seguintes variáveis de entrada para o sistema fuzzy de controle de irrigação: i) Temperatura do ambiente (T) $\left(0\right.$ a $\left.50{ }^{\circ} \mathrm{C}\right)$; ii) Umidade do ar (H) (0 a 100\%); iii) Umidade do solo (M) (0 a 100\%); iv) Saldo na carteira (B) (0 a 120). Como variável de saída foi definida: i) Tempo para o acionamento da bomba de água (P) (0 a 7 segundos). A Tabela I mostra as funções de pertinência usadas para definição dos conjuntos fuzzy. Para o saldo, foram definidos três predicados (Baixo, Médio e Alto) tendo como valor médio a quantidade para manter a irrigação da horta por uma semana. As regras definidas sobre os predicados das variáveis de umidade do ar (Alta e Baixa), temperatura do ambiente (Fria e Quente) e umidade do solo (Seca, Moderada e Molhada) as características do orégano. Os predicados do tempo de bombeamento (Muito Curto, Curto, Médio e Longo) consideram o tempo de acionamento médio para irrigar a quantidade de água exigida pelas plantas em um período de 6 horas $(50 \mathrm{ml})$.

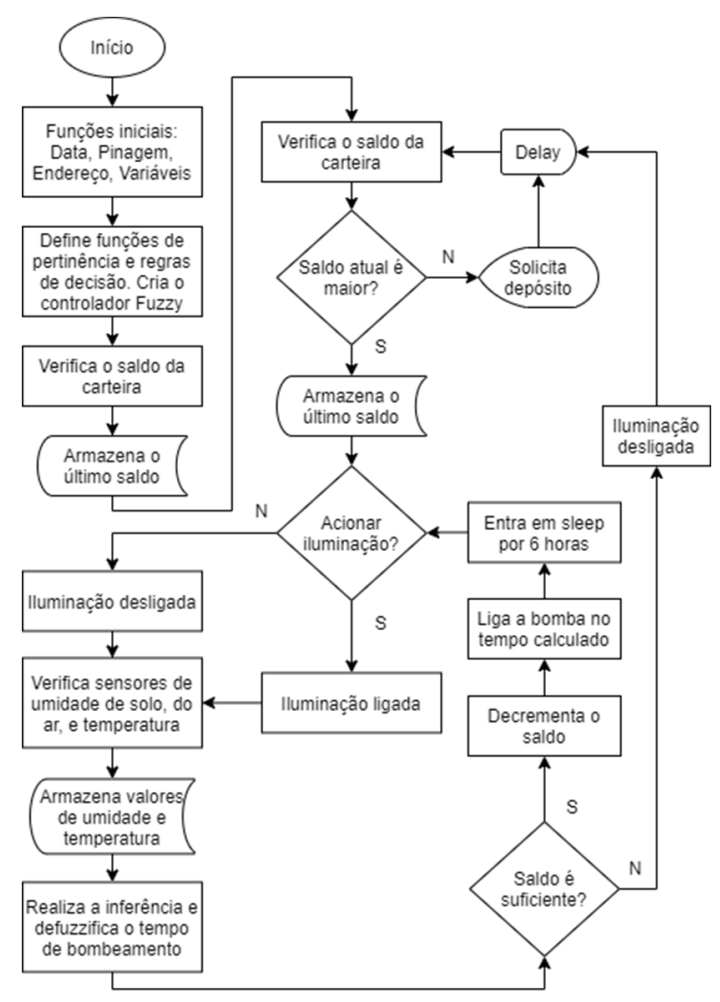

Fig. 2. Fluxograma do sistema com a lógica fuzzy inclusa

TABELA I. FUNÇÕES DE PERTINÊNCIA.

\begin{tabular}{|c|c|}
\hline$\mu_{B}^{\text {Baixo }}(x)=0, \forall x \geq 45$ & $\mu_{M}^{\text {Seco }}(x)=0, \forall x \geq 40 \%$ \\
$\mu_{B}^{\text {Baixo }}(x)=1, \forall x \leq 25$ & $\mu_{M}^{\text {Seco }}(x)=1, \forall x \leq 30 \%$ \\
\hline$\mu_{B}^{\text {Médio }}(x)=0, \forall x \leq 25$ & $\mu_{M}^{\text {Mod. }}(x)=0, \forall x \leq 30 \%$ \\
$\& x \geq 95$ & $\& x \geq 70 \%$ \\
$\mu_{B}^{\text {Médio }}(x)=1, \forall 45 \leq x \leq 75$ & $\mu_{M}^{\text {Mod. }}(x)=1, \forall 40 \% \leq x \leq 60 \%$ \\
\hline$\mu_{B}^{\text {Much }}(x)=0, \forall x \leq 75$ & $\mu_{M}^{\text {Molhada }}(x)=0, \forall x \leq 60 \%$ \\
$\mu_{B}^{\text {Much }}(x)=1, \forall x \geq 95$ & $\mu_{M}^{\text {Molhada }}(x)=1, \forall x \geq 70 \%$ \\
\hline$\mu_{H}^{\text {Baixo }}(x)=0, \forall x \geq 75 \%$ & $\mu_{T}^{\text {Fria }}(x)=0, \forall x \geq 34^{\circ} \mathrm{C}$ \\
$\mu_{H}^{\text {Baixo }}(x)=1, \forall x \leq 60 \%$ & $\mu_{T}^{\text {Fria }}(x)=1, \forall x \leq 22^{\circ} \mathrm{C}$ \\
\hline$\mu_{H}^{\text {Alto }}(x)=0, \forall x \leq 65 \%$ & $\mu_{T}^{\text {Quente }}(x)=0, \forall x \leq 22^{\circ} \mathrm{C}$ \\
$\mu_{H}^{\text {Alto }}(x)=1, \forall x \geq 80 \%$ & $\mu_{T}^{\text {Quente }}(x)=1, \forall x \geq 34^{\circ} \mathrm{C}$ \\
\hline$\mu_{P}^{\text {M.Curto }}(x)=0, \forall x \geq 2 s$ & $\mu_{P}^{\text {Médio }}(x)=0, \forall x \leq 3 s$ \\
$\mu_{T}^{\text {M.Curto }}(x)=1, \forall x \leq 1 s$ & $\& x \geq 6 s$ \\
$\mu_{P}^{\text {Curto }}(x)=0, \forall x \leq 1 s$ & $\mu_{T}^{\text {Médio }}(x)=1, \forall 4 s \leq x \leq 5 s$ \\
$\& x \geq 4 s$ & $\mu_{P}^{\text {Longo }}(x)=0, \forall x \leq 5 s$ \\
$\mu_{T}^{\text {Curto }}(x)=1, \forall 2 s \leq x \leq 3 s$ & $\mu_{T}^{\text {Longo }}(x)=1, \forall x \geq 6 s$ \\
\hline
\end{tabular}


TABELA II. BASE DE REGRAS PARA O CONTROLE DA IRRIGAÇÃO.

\begin{tabular}{|c|c|c|c|}
\hline SE & Umidade do ar é Alta & SE & Umidade do ar é Baixa \\
\hline E & Temperatura do ar é Fria & E & Temperatura do ar é Quente \\
\hline E & Umidade do solo é Molhada & E & Umidade do solo é Molhada \\
\hline E & Saldo é Alto & E & Saldo é Baixo \\
\hline ENTÃO & Bombeamento é Muito Curto & ENTÃO & Bombeamento é Curto \\
\hline SE & Umidade do ar é Alta & SE & Umidade do ar é Baixa \\
\hline E & Umidade do solo é Moderada & E & Umidade do solo é Moderada \\
\hline E & Saldo é Baixo & E & Saldo é Baixo \\
\hline ENTÃO & Bombeamento é Curto & ENTÃO & Bombeamento é Médio \\
\hline SE & Umidade do ar é Alta & SE & Umidade do ar é Baixa \\
\hline E & Umidade do solo é Molhada & E & Umidade do solo é Molhada \\
\hline E & Saldo é Médio & E & Saldo é Médio \\
\hline ENTÃO & Bombeamento é Muito Curto & ENTÃO & Bombeamento é Curto \\
\hline SE & Umidade do ar é Alta & SE & Umidade do ar é Baixa \\
\hline E & Umidade do solo é Seca & E & Umidade do solo é Seca \\
\hline E & Saldo é Médio & E & Saldo é Médio \\
\hline ENTÃO & Bombeamento é Médio & ENTÃO & Bombeamento é Longo \\
\hline SE & Umidade do solo é Molhada & SE & Umidade do solo é Molhada \\
\hline E & Saldo é Alto & E & Saldo é Baixo \\
\hline ENTÃO & Bombeamento é Curto & ENTÃO & Bombeamento é Muito Curto \\
\hline SE & Umidade do solo é Moderada & SE & Umidade do solo é Seca \\
\hline E & Saldo é Alto & E & Saldo é Baixo \\
\hline ENTÃO & Bombeamento é Médio & ENTÃO & Bombeamento é Médio \\
\hline SE & Umidade do solo é Seca & SE & Umidade do solo é Moderada \\
\hline E & Saldo é Alto & E & Saldo é Médio \\
\hline ENTÃO & Bombeamento é Longo & ENTÃO & Bombeamento é Médio \\
\hline
\end{tabular}

\section{EXPERIMENTOS E ANÁLISE DE DESEMPENHO}

Inicialmente, as condições de data e hora, a pinagem a ser utilizada no Raspberry, e a biblioteca PyOTA são carregadas. Uma função de verificação do saldo da carteira utilizada é implementada, e será utilizada para as consultas de saldo feitas enquanto o sistema estiver rodando. A Figura 3 mostra o experimento em funcionamento.

O endereço da carteira utilizada é associado à horta, onde são feitos os recebimentos de criptomoeda. Ao se rodar o sistema, as diferenças de saldo positivas verificadas na carteira possibilitam a iluminação artificial e o loop de verificação de umidade e consequente irrigação, se necessário. Um QR code pode ser associado ao endereço IOTA, facilitando as transferências feitas por outras carteiras instaladas em smartphones. Enquanto a carga estiver acionada, caso haja um novo acréscimo de saldo, o tempo de funcionamento da horta se estende proporcionalmente a este acréscimo. Ao se consumir a diferença de saldo, a horta se desativa, e o processo continua em um loop de verificação de saldo e acionamento e desligamento da horta.

É evidente que o tempo que a rede demora para aprovar e mostrar a transação na carteira de destino da transferência como saldo, é importante para a proposta deste artefato. Dessa forma, para as medições de tempo de transação foi utilizada a seguinte metodologia: duas carteiras IOTA foram criadas, ambas utilizando a Wallet Trinity, recomendada pela Fundação IOTA à época. Uma das carteiras foi associada à horta, para receber os pagamentos. A outra carteira seria um cliente qualquer da horta, que faz os pagamentos. Na carteira, além do valor monetário transferido, é possível também associar mensagens de texto, onde o cliente pode se identificar e especificar o pagamento também. A carteira recebedora consegue ler essas informações. Outra informação interessante é que os tempos medidos se referem a toda a transação, de ponta a ponta, ou seja, do momento em que o cliente pressiona o 'Enviar' de sua carteira até o momento em que a carteira recebedora acusa o recebimento. É uma análise do ponto de vista dos usuários da rede. Foram feitas 100 medições de tempo de confirmação de transação, em um período de três dias, de 12 de janeiro a 14 de janeiro de 2021, em horários distintos, que podem ser vistas no gráfico da Figura 4.

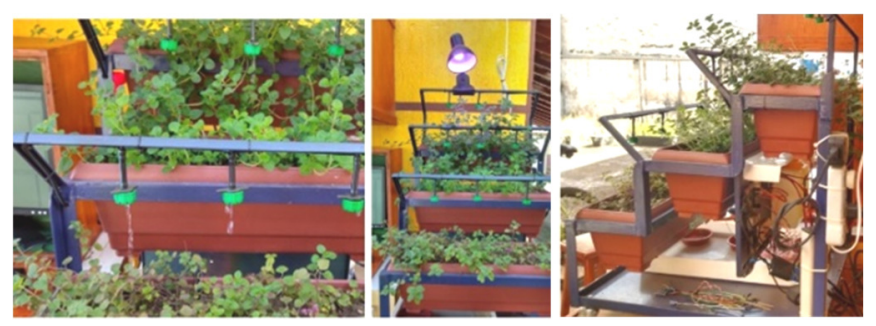

Fig. 3. Experimento em funcionamento.

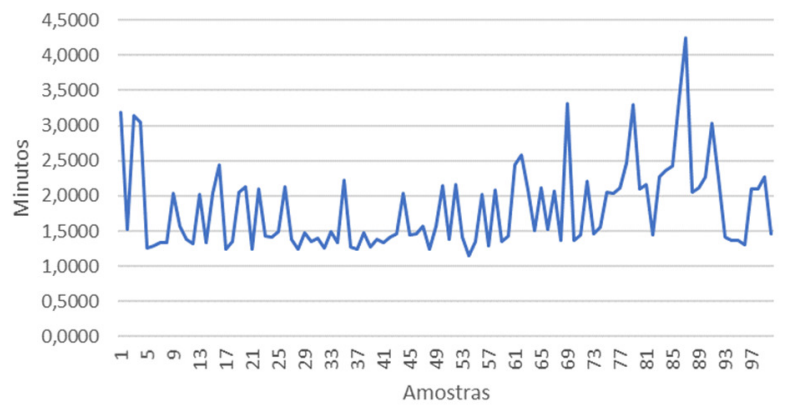

Fig. 4. Tempo de confirmação de transação em janeiro de 2021.

O tempo médio de confirmação de transação para o endereço em uso foi de 2,02 minutos. Para um nível de confiança de $95 \%$, e com um desvio padrão de 0,57 minutos $^{2}$, a margem de erro foi de 0,13 minutos, ficando o intervalo de confiança da medida entre 1,89 a 2,15 minutos. Do ponto de vista dos usuários, estas medições são bem realistas, e trazem uma boa noção de como a aplicação dos usuários (no caso, a horta) vai demorar para reagir aos pagamentos recebidos.

O nó da rede ao qual o Raspberry Pi está conectado também pode influenciar no tempo de confirmação da transação, dependendo do grau de atividade do nó, da quantidade de TIPs deste nó, quantidade de vizinhos, etc. Em teoria, o tempo de confirmação é de 2 milissegundos [15], mas as medições do próprio Tangle, conforme a figura 4 acima, mostram que na prática isso ainda não acontece. Nos casos em que a transação demore muito tempo para ser confirmada, ela precisa ser recolocada ou referenciada explicitamente em outra transação (transação vazia). À medida em que mais transações ocorrem, mais rapidamente ocorrerão as confirmações, pois elas serão mais rapidamente confirmadas pelas transações subsequentes. A rigor, o limite de velocidade de confirmação de transação é tão somente o limite físico da velocidade da rede. Com o crescimento da rede IOTA, existirão mais dispositivos, que acelerarão o processo de confirmação da transação, ficando a rede cada vez mais rápida. 
O sistema de controle fuzzy foi implementado na linguagem Python, utilizando a biblioteca Scikit-fuzzy. Para analisar o funcionamento do módulo de irrigação automática, foi realizada uma simulação de uma semana em que um deposito inicial de 64 IOTAs foi realizado. Os valores de temperatura e umidade do ar para a simulação foram extraídos do banco de dados meteorológicos do INPE. Os dados são da cidade de Maria da Fé, MG, cidade a $35 \mathrm{~km}$ de Santa Rita do Sapucaí, para o período de 19 a 26 de abril de 2021. A umidade do solo foi configurada inicialmente com o valor de $50 \%$ para uma amostra de solo de $2,250 \mathrm{~kg}$. A quantidade de água no solo foi decrementada pelo valor da evapotranspiração horária calculada por uma adaptação da equação Penman-Monteith. A umidade do solo foi incrementada de acordo com o tempo de acionamento da bomba decidido pelo controlador. A figura 5 mostra a umidade do solo variando no tempo. Pode-se observar que o controlador consegue manter um nível adequado para o desenvolvimento das plantas, compensando a evapotranspiração. A média dos valores da umidade do solo simulados foi de $68.15 \%$, restando 9 IOTAs no saldo.

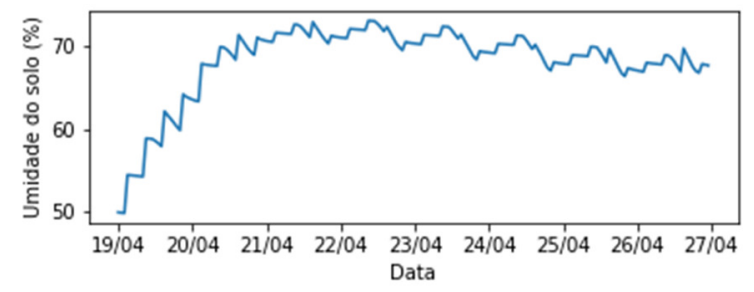

Fig. 5. Controle da Umidade do Solo realizado pelo sistema fuzzy.

\section{CONCLUSÕES}

Este artigo apresenta uma prova de conceito experimental da aplicação de micropagamentos em moeda digital IOTA em uma pequena horta urbana, com decisões baseadas nos princípios da lógica difusa, podendo perfeitamente ser utilizada em pequeníssimos dispositivos de IoT, a custo baixo, e também com pouco consumo de energia. Melhorias na codificação e hardwares mais sofisticados podem ser implementados, a partir desta ideia inicial, para atingir objetivos maiores, e atender a todo tipo de aplicação de acionamentos mediante pagamentos digitais. Outros sensores podem futuramente ser acoplados para melhorar o suporte na tomada decisão do cuidador da horta ou do módulo de controle fuzzy, como sensor de nível para o tanque de água, sensor de vazão e sensor de exposição à luz, por exemplo. Com isso, poderiam ser definidas regras para outros acionadores, como a iluminação artificial. Experimentos com o sistema fuzzy no Raspberry devem ser realizados para verificar o funcionamento com dados reais.

Em alguns casos de aplicações reais, o tempo de confirmação da transação ainda pode ser considerado como um gargalo para o uso. Porém à medida em que a quantidade de dispositivos conectados à rede crescer, mais rápida ficará a confirmação da transação, e mais rapidamente poderá ser acionada a carga conectada ao artefato.

Um outro desenvolvimento importante, que pode ser considerado como uma futura sequência deste trabalho, é usar um dispositivo físico, tal como o próprio Raspberry $\mathrm{Pi}$, para gravar e ler dados no IOTA Tangle. Assim, todos os dados de sensoriamento do serviço de IoT, pago à distância, poderão ser verificados pelo pagador, inclusive com a possibilidade de gravação de imagens no Tangle. Além disso, uma plataforma online para consumir esses dados poderia ser desenvolvida e usada para monitoramento remoto do estado atual da horta e para consulta do histórico das medidas dos sensores e transações realizadas. O módulo de irrigação automática também se beneficiaria com a consulta de dados históricos, podendo considerar a variação das condições ambientais no tempo e tomar decisões mais precisas.

A IOTA foi utilizada nesse experimento, mas existem outras criptomoedas que se propõem ao uso em micropagamentos de IoT, como por exemplo a IoT Chain - ITC, de origem chinesa. Seria também um trabalho futuro bastante interessante fazer a análise de desempenho desta criptomoeda, estabelecendo comparações com a IOTA, em termos de desempenho. O consumo de energia do sistema também é um fator a ser considerado em trabalhos futuros.

\section{AGRADECIMENTOS}

Este trabalho foi parcialmente financiado pela RNP, com recursos do MCTI, processo $\mathrm{N}^{\circ}$ 01245.010604/2020-14, sob o projeto Sistemas de Comunicações Móveis de $6^{\mathrm{a}}$ Geração (6G) do Centro de Referência em Radiocomunicações (CRR) do Instituto Nacional de Telecomunicações - Inatel, Brasil.

\section{REFERÊNCIAS}

[1] Vicente, R.J., A Criptomoeda como método alternativo para realizar transações financeiras. Revista Maiêutica, Indaial, v. 2, n. 01, p 85-94, 2017. ISSN 2525-8397, 2017

[2] Biryukov, A., \& Pustogarov, I., Proof-of-work as anonymous micropayment: Rewarding a Tor relay. In International Conference on Financial Cryptography and Data Security (pp. 445-455). Springer, Berlin, Heidelberg, 2015

[3] Popov, S., The tangle. https://iota. org/IOTA_Whitepaper, 2017

[4] Popov, S., Saa, O., \& Finardi, P., Equilibria in the Tangle. Computers \& Industrial Engineering, 136, 160-172, 2019

[5] Zadeh, L. A., Fuzzy logic. Computer, 21(4), 83-93, 1988

[6] Divya, M., \& Biradar, N. B., IOTA-Next Generation Blockchain. International Journal of Engineering and Computer Science, 7(04), 23823-23826, 2018

[7] Hu, J., IOTA Tangle: Introductory overview of White paper for Beginners. 2017. Disponível em <https://medium.com>, acessado em fevereiro 2020.

[8] Raspberrypi. Disponível em: <https://www.raspberrypi.org/downloads/raspbian/>. Acessado em fevereiro 2021.

[9] GitHub. Disponível em <https://github.com/iotaledger/iota.py>. Acessado em fevereiro 2021

[10] GitHub Gist. Disponível em: <https://gist.github.com/huggre/a3044e6094867fe04096e0c64dc60f3b>. Acessado em novembro 2020.

[11] TheTangle. Disponível em: <https://thetangle.org/statistics/popularaddresses $>$. Acessado em março 2020.

[12] Alomar, B., \& Alazzam, A., A smart irrigation system using IoT and fuzzy logic controller. In 2018 Fifth HCT Information Technology Trends (ITT) (pp. 175-179). IEEE. 2018.

[13] Abdullah, N., Durani, N. A., Shari, M. F., Soon, S. K., Hau, V. K. W., Siong, W. N., \& Ahmad, K. A., Towards Smart Agriculture Monitoring Using Fuzzy Systems. IEEE Access. 2020.

[14] Tanglemonitor. Disponível em <https://tanglemonitor.com/ $>$. Acessado em Março 2020.

[15] IOTA Stack Exchange. Disponível em: <https://iota.stackexchange.com/questions/88/what-is-the-averagetransaction-time-in-iota>. Acessado em março 2020. 